\title{
Uso do laser diodo para correção cirúrgica de estenose de narina e prolongamento de palato mole em cão portador da síndrome do braquicéfalo - relato de caso
}

Use of laser diode for surgical correction of stenosis of nostrils and elongated soft palate in dog with brachycephalic syndrome - case report

Uso de láser de diodo para la corrección quirúrgica de la estenosis de las fosas nasales y la prolongación del paladar blando en un perro con síndrome braquicefálico: reporte de caso

Aguinaldo Francisco Mendes Junior ORCID: https://orcid.org/0000-0002-2780-9294 Universidade Federal Fluminense, Brasil E-mail: aguinaldo_zootec@hotmail.com

Marina Ribeiro Hotz

ORCID: https://orcid.org/0000-0001-9283-8721 Universidade Federal Fluminense, Brasil E-mail: marinahotz@id.uff.br

Pâmela Cristina De Souza Duarte ORCID: https://orcid.org/0000-0001-7565-1519 Universidade Federal Fluminense, Brasil E-mail: pameladuarte2@gmail.com

Gabriela Souza Leão Bezerra Dos Santos ORCID: https://orcid.org/0000-0002-2454-573X Universidade Federal Fluminense, Brasil E-mail: gabriellaleao@id.uff.br Asy Pepe Sanches Neto ORCID: https://orcid.org/0000-0001-6883-6961 Universidade Federal Fluminense, Brasil E-mail: Asy.sanches@gmail.com

Gabriele Barros Mothé

ORCID: https://orcid.org/0000-0003-0835-5239 Universidade Federal Fluminense, Brasil E-mail: gabimothe2@hotmail.com

Ana Maria Barros Soares ORCID: https://orcid.org/0000-0001-8757-1946 Universidade Federal Fluminense, Brasil E-mail: anasoaresvet@gmail.com Nádia Regina Pereira Almosny ORCID: https://orcid.org/0000-0001-7043-0717 Universidade Federal Fluminense, Brasil E-mail: nadiaalmosny@id.uff.br

\begin{abstract}
Resumo
Inúmeras deformações obstrutivas de etiologia congênita compõem a síndrome braquicefálica. Dentre elas destacamse a estenose de narinas e o prolongamento de palato mole, para os quais são de grande importância o diagnóstico e o tratamento cirúrgico precoce, visando a minimizar a progressão da doença e o iminente risco de óbito do paciente. Este trabalho objetiva relatar o emprego do laser diodo de alta potência na correção cirúrgica da estenose de narina e do prolongamento de palato mole em um cão braquicefálico de um ano de idade com quadro clínico de dispneia inspiratória, ronco, intolerância ao exercício e síncope. O animal foi submetido à rinoplastia e palatoplastia, apresentando significativa melhora clínica após os procedimentos. Isso mostra que o emprego do laser de diodo de alta potência é eficaz no tratamento cirúrgico da síndrome braquicefálica, proporcionando bons resultados funcionais e estéticos e, por conseguinte, melhora no bem-estar e na qualidade de vida do animal.
\end{abstract}

Palavras-chave: Estafilectomia; Qualidade de vida; Rinoplastia.

\begin{abstract}
Various obstructive deformations of congenital etiology make up the brachycephalic syndrome, among them stenosis of the nostrils and prolongation of the soft palate. Early diagnosis and surgical treatment are of great importance, in order to minimize the disease progression and the imminent risk of death for the patient. This paper aims to report the use of diode laser in the surgical correction of stenosis of the nostrils and soft palate prolongation in a one-year-old
\end{abstract}


brachycephalic dog with clinical symptoms of inspiratory dyspnea, snoring, exercise intolerance and syncope. The animal underwent rhinoplasty and palatoplasty with diode laser, and showed significant clinical improvement after the procedures, proving that the use of the diode laser is effective in the treatment of brachycephalic syndrome, bringing good functional and aesthetic results and improving well-being and quality of life of the animal.

Keywords: Staphlectomy; Quality of life; Rhinoplasty.

\section{Resumen}

Numerosas deformaciones obstructivas de etiología congénita componen el síndrome braquicefálico. Entre ellos, destacamos la estenosis de las fosas nasales y la prolongación del paladar blando, por lo que el diagnóstico precoz y el tratamiento quirúrgico son de gran importancia, con el fin de minimizar la progresión de la enfermedad y el riesgo inminente de muerte del paciente. Este trabajo tiene como objetivo reportar el uso de láser de diodo de alta potencia en la corrección quirúrgica de la estenosis de las fosas nasales y la prolongación del paladar blando en un perro braquicefálico de un año con síntomas clínicos de disnea inspiratoria, ronquidos, intolerancia al ejercicio y síncope. El animal fue sometido a rinoplastia y palatoplastia, mostrando una mejora clínica significativa después de los procedimientos. Esto demuestra que el uso de láser de diodo de alta potencia es eficaz en el tratamiento quirúrgico del síndrome braquicefálico, proporcionando buenos resultados funcionales y estéticos y, por tanto, mejora el bienestar y la calidad de vida del animal.

Palabras clave: Estafilectomía; Calidad de vida; Rinoplastia.

\section{Introdução}

Apesar dos problemas relacionados à saúde, ao bem-estar e à qualidade de vida, há em todo o mundo um aumento expressivo do número de cães pertencentes às raças braquicefálicas. Esses animais, devido à seleção genética, apresentam cada vez mais anormalidades anatômicas obstrutivas em vias aéreas anteriores, gerando importantes sinais clínicos que caracterizam a síndrome braquicefálica ou síndrome dos braquicéfalos (Packer \& Tivers, 2015; Crane, Rozanski, Abelson, Laforcade, 2017; Packer, O’Neill, Fletcher, Farnworth, 2019).

Na síndrome dos braquicéfalos, as modificações anatômicas causam obstrução das vias aéreas anteriores e impedem o fluxo adequado do ar até os pulmões, aumentando o esforço inspiratório, a pressão negativa e o turbilhonamento do ar nessas regiões. As alterações classificadas como primárias são: estenose de narina, prolongamento de palato mole, cornetos nasais aberrantes, macroglossia e hipoplasia de traqueia. Esses defeitos podem provocar alterações secundárias, como eversão de sáculos laríngeos, edema e inflamação de nasofaringe, paralisia e colapso de laringe, e hipertensão pulmonar (Dupré \& Heidenrich, 2016).

A realização precoce dos tratamentos clínico e cirúrgico é de extrema importância para a melhoria da qualidade de vida e o bem-estar dos animais afetados pela síndrome braquicefálica, além da diminuição do risco iminente de óbito (Lodato \& Hedlund, 2012).

A fim de diminuir a resistência e facilitar o fluxo do ar pelas vias aéreas anteriores, a correção cirúrgica das deformidades anatômicas que causam as diferentes obstruções mecânicas é a principal indicação para o correto tratamento e melhor resposta clínica a essa enfermidade (Trappler \& Moore, 2011, Dupré \& Heidenrich, 2016), com mínimo risco para o paciente (Tarricone, Hayes, Singh, Davis, 2019).

Novas técnicas de correção cirúrgica estão sendo implementadas no tratamento da síndrome dos cães braquicefálicos, como uso de laser cirúrgico (Emmerson, 2014). Há inúmeras vantagens no emprego desse artifício em comparação com os procedimentos cirúrgicos convencionais (Dunié-mérigot, Bouvy, Poncet, 2010; Keats, 2012; Lodato \& Mauterer, 2014).

O laser diodo interage com o tecido alvo e além de realizar a ablação, tem ação bioestimuladora, sela vasos sanguíneos, linfáticos e terminações nervosas. Desta forma propicia uma melhor hemostasia, diminui a necessidade da realização de suturas, encurtando o tempo cirúrgico. No trans e pós-operatório, constata-se um índice menor de edema, inflamação e dor (Eeg, 2003; Dunié-mérigot, et al., 2010).

Frente ao exposto, o presente estudo objetiva relatar a técnica e os resultados pós-cirúrgicos obtidos com o uso do laser na realização de rinoplastia e palatoplastia como tratamento da síndrome do cão braquicefálico. 


\section{Metodologia}

Este artigo trata-se de um relato de caso, abordado de forma descritiva e qualitativa, onde segundo Pereira et al, (2018), caracteriza- se como uma pesquisa que por via direta recolhe dados relativos ao estudo através do acesso aos registros médicos e aos exames fornecidos, sendo o pesquisador o instrumento primordial.

$\mathrm{O}$ paciente deste relato foi avaliado clinicamente e submetido a exames complementares que garantiram segurança e bem estar do animal, indicando que o mesmo estava apto a passar pelos procedimentos cirúrgicos e anestésicos, sendo então encaminhado para cirurgia após a assinatura pelos respectivos tutores de Termo de Consentimento Livre e Esclarecido, no qual constavam descrições adequadas e informações essenciais ao entendimento da cirurgia, anestesia e pós-operatório do animal.

\section{Relato de Caso}

Foi atendido no Hospital Veterinário Universitário Firmino Mársico Filho, da Universidade Federal Fluminense (HUVET-UFF), na cidade de Niterói-RJ, um canino, macho inteiro, da raça Buldogue francês, de três anos de idade. O paciente apresentava intensa dispneia inspiratória padrão obstrutivo, ronco acordado e ao dormir, grave intolerância ao exercício, acompanhada de cianose e episódios de síncope em atividade física.

No exame clínico, observou-se o paciente ofegante, com mucosas levemente hiperêmicas, hidratação normal, temperatura retal $38,7^{\circ} \mathrm{C}$, frequência cardíaca de $120 \mathrm{bpm}$, sem alteração na ausculta cardíaca, e sons pulmonares aumentados. Em função dos sinais clínicos e da predisposição racial, estabeleceu-se a suspeita clínica de síndrome braquicefálica.

Foi realizada inspeção direta das narinas, bem como a classificação do grau de obstrução de acordo com os parâmetros de Liu et al., (2017), que determinaram como "narinas abertas" aquelas que se apresentam bem abertas e cuja parede lateral não toca a medial; "narinas levemente estenosadas" as que se apresentam levemente fechadas, mas cuja parede lateral ainda não toca a medial; "narinas moderadamente estenosadas" aquelas cuja parede lateral toca a parede medial da narina na porção dorsal e a porção caudal se mantém aberta; e "narinas gravemente estenosadas" aquelas cuja parede lateral toca a parede medial da narina na porção dorsal e na porção caudal. Com base nesse critério, concluiu-se que o paciente apresentava estenose grave de narina, confirmando o diagnóstico (Figura 1) e sendo então indicadas a rinoplastia e a laringoscopia da região de orofaringe, para avaliação do palato mole, e a correção cirúrgica em caso de prolongamento. Para a avaliação pré-cirúrgica foram realizadas análise hematológica, bioquímica sérica (dosagem de ureia, creatinina, glicose, proteínas totais, albumina, globulina, fosfatase alcalina, alanina-aminotransferase (ALT), aspartatoaminotransferase (AST)); avaliação eletrocardiográfica e ecocardiográfica; além de exame radiográfico da região do tórax. Após a análise dos resultados, o animal foi liberado para a realização do procedimento cirúrgico. 
Figura 1. Cão da raça Buldogue francês, três anos de idade, apresentando estenose bilateral de narinas grave.



Fonte: Projeto Narizinho - UFF (2019).

No protocolo anestésico, utilizou-se como medicação pré-anestésica nalbufina $(0,5 \mathrm{mg} / \mathrm{kg})$ por via intramuscular; na indução, o animal recebeu propofol $(3 \mathrm{mg} / \mathrm{kg})$ associado a midazolam $(0,3 \mathrm{mg} / \mathrm{kg})$ intravenoso; neste momento, foi realizada a laringoscopia e constatado o prolongamento do palato mole. A manutenção foi realizada pela técnica inalatória utilizando isofluorano $1,5 \%$. Durante o período transcirúrgico, foi administrada, na forma intravenosa, dipirona na dose de $25 \mathrm{mg} / \mathrm{kg}$ para analgesia e hidrocortisona $(10 \mathrm{mg} / \mathrm{kg})$ como anti-inflamatório.

Após a assepsia da região cirúrgica, o paciente foi posicionado em decúbito ventral com a cabeça levemente levantada, e a área facial foi coberta por um pano de campo, evidenciando apenas a região da narina. Para os procedimentos, utilizou-se aparelho DMC Vetlase ${ }^{\circledR}$ com laser diodo e comprimento de onda laser infravermelho $808 \mathrm{~nm} \pm 10 \mathrm{~nm}$ e potência útil de $9 \mathrm{~W} \pm 20 \%$, com fibra de diâmetro $400 \mu \mathrm{m}$.

$\mathrm{Na}$ rinoplastia, foi realizada a técnica de alavestibuloplastia, utilizou-se o modo contínuo na potência de $2,8 \mathrm{~W}$, procedendo-se à marcação interna e externa das asas das narinas a serem retiradas (Figura 2A, B, C, D), e, em seguida, fez-se a incisão pela porção medial até a porção caudal da asa hipertrofiada e, posteriormente, um corte perpendicular em profundidade, liberando assim a abertura da cavidade nasal (Figura 2E, F). 
Figura 2. Realização de rinoplastia com laser diodo de alta potência em canino da raça Buldogue francês. Marcações das áreas a serem incisadas (A, B, C, D). Realização da fotoablação das porções externa (E) e interna (F).

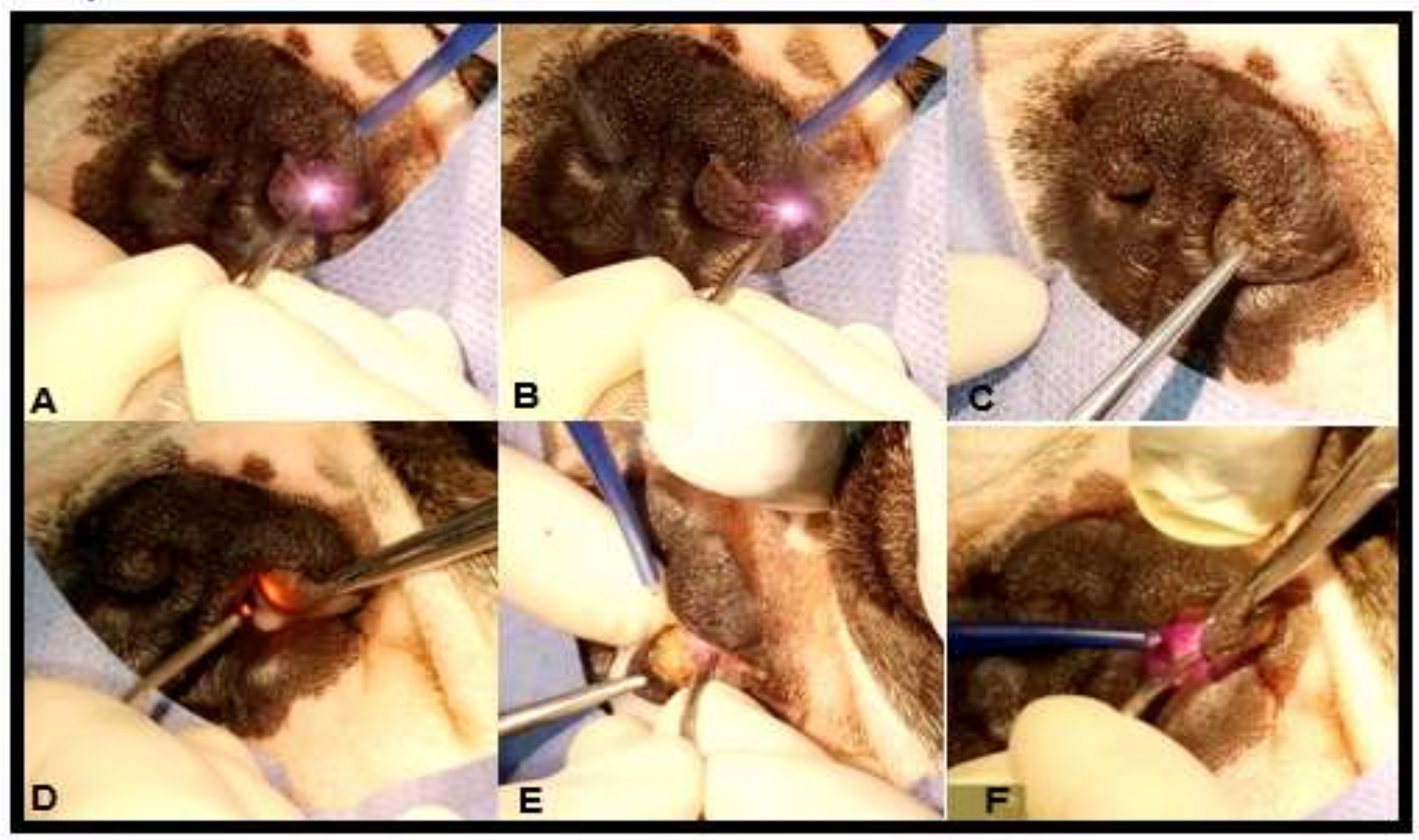

Fonte: Projeto Narizinho UFF (2019).

O procedimento foi realizado bilateralmente, dispensando cuidado à proteção do efeito térmico do laser nas regiões adjacentes às narinas, mediante o uso de algodão embebido em soro fisiológico. A realização da ablação mostrou-se bem eficaz e não houve sangramento (Figura 3A). Após 30 dias, as narinas apresentaram aspecto estético e funcional considerados satisfatórios (Figura 3B).

Figura 3. Aspecto das narinas de canino da raça Buldogue francês imediatamente após a fotoablação com laser diodo para rinoplastia com a técnica da alavestibuloplastia (A) e 30 dias após o procedimento (B).

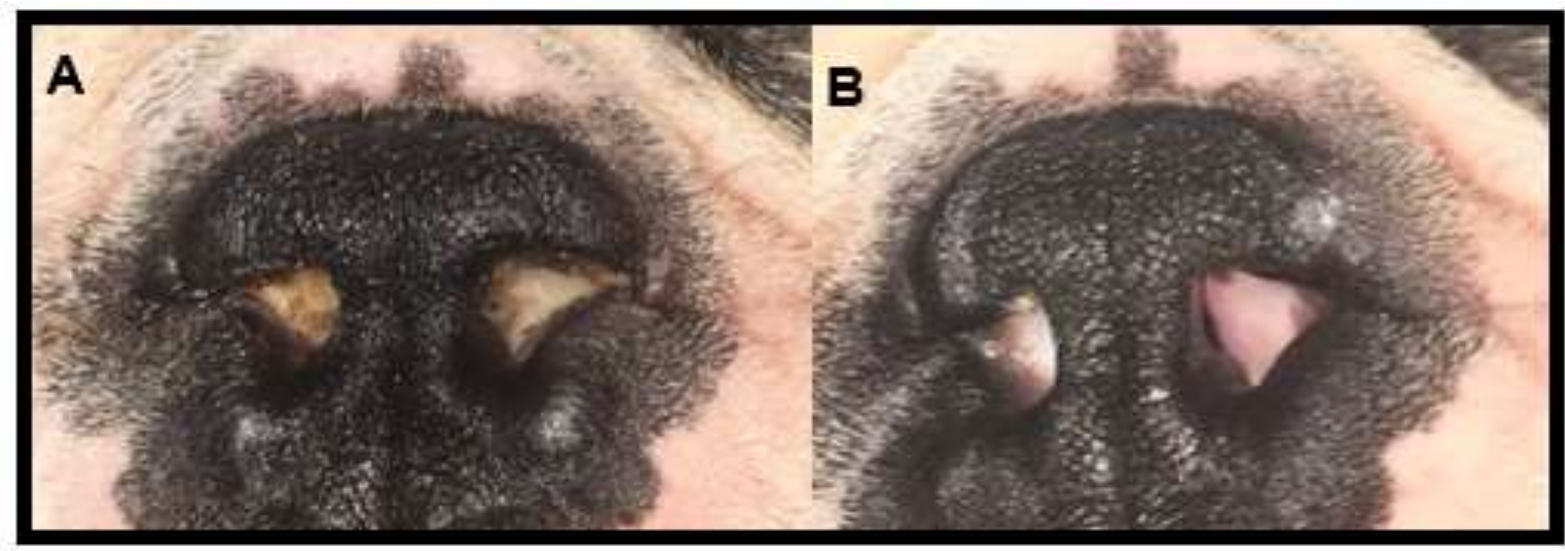

Fonte: Projeto Narizinho UFF (2019). 
A ressecção cirúrgica do palato mole alongado ou palatoplastia também foi realizada no paciente em questão utilizando o mesmo aparelho anteriormente especificado. Com o animal ainda em decúbito ventral, procedeu-se à abertura da boca, empregando-se um "abre-bocas”, para visualização e acesso à região de orofaringe.

A palatoplastia teve início com o animal em posicionamento anatômico normal e emprego de três pontos de reparo nas extremidades do palato mole para facilitar a identificação; a marcação da linha de incisão foi realizada por meio de pequenas marcações com o laser (Figura 4). Tomou-se como base a região de encontro do palato mole com a porção caudal das tonsilas palatinas e a ponta da epiglote. Após a marcação, as áreas adjacentes foram protegidas com gaze embebida em soro fisiológico. Realizou-se a exposição da estrutura e a incisão com o laser diodo no modo contínuo, na potência de 3,5W, até a retirada total da porção alongada do palato mole.

Figura 4. Exposição e marcação a laser do palato mole alongado em cão da raça Buldogue francês, três anos (A). Pósoperatório imediatamente após a fotoablação com laser diodo palatoplastia (B).

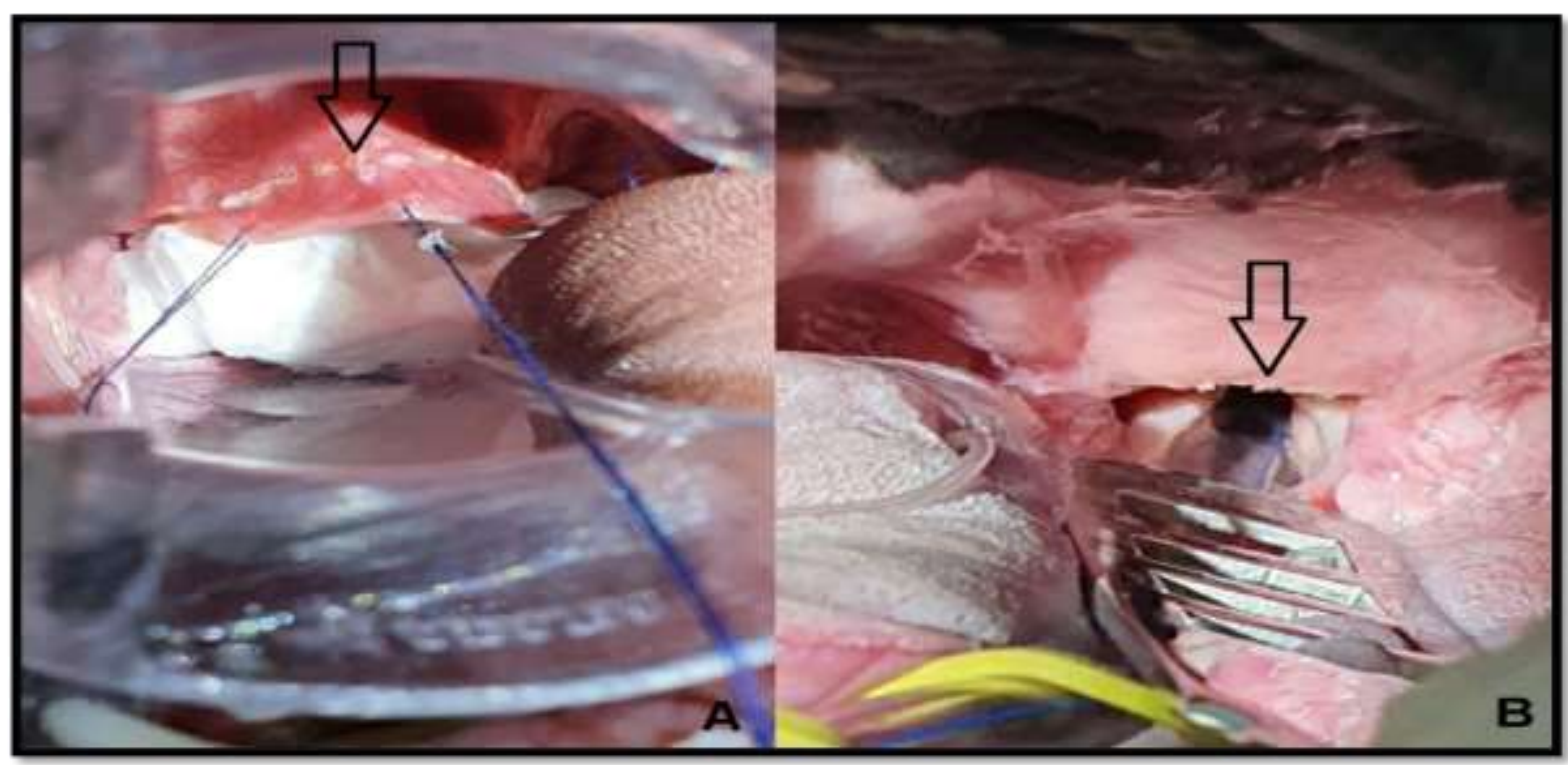

Fonte: Projeto Narizinho UFF (2019).

\section{Discussão}

Inúmeras deformações obstrutivas de etiologia congênita compõem a síndrome braquicefálica, entre elas a estenose bilateral de narina e o prolongamento de palato mole (Dupré, 2016; De Lorenzi, Bertoncello, Montovani, 2018), alterações características apresentadas pelo paciente, que denotam grande potencial obstrutivo, podendo levar à progressão da doença. Ao exame clínico foi observado importante grau de obstrução das narinas o que, associado à sintomatologia clínica do paciente, sustentou o diagnóstico de síndrome braquicefálica. É sabido que animais portadores de estenose de narina apresentam significativa redução no espaço aéreo, o que dificulta a chegada do ar até os pulmões (Torrez \& Hunt, 2006; Oechtering, 2010). Por ser a alteração anatômica mais anterior, a estenose de narina pode ser considerada um dos principais pontos de obstrução das vias aéreas, causando importante sobrecarga inspiratória (Lodato \& Hedlund, 2012).

A laringoscopia realizada no animal anestesiado mostrou expressiva obstrução da nasofaringe pelo palato alongado, alteração primária de alta prevalência na síndrome dos braquicéfalos (Slawuta, Glińska-Suchocka, Cekiera, 2015). Durante a inspiração, no momento em que o ar é recrutado pela cavidade oral, o palato mole alongado reduz o lúmen da nasofaringe e da orofaringe, aumentando a resistência do fluxo do ar pelas vias aéreas (Pichetto, Arrighi, Roccabianca \& Romussi, 2011). 
O cão apresentava dispneia inspiratória, ronco, intolerância ao exercício e síncope, sinais clínicos característicos dessa enfermidade (Emmerson, 2014; Packer, Hendricks, Tivers, 2015; Liu et al., 2017). A sintomatologia se desenvolve em conformidade com a fisiopatologia da doença, e sua gravidade varia de acordo com o nível de obstrução das vias aéreas anteriores (Oechtering, 2010; Packer \& tivers, 2015; Ladlow, Liu, Kalmar \& Sargan, 2018). Conforme foi relatado, em função dos sinais clínicos, o paciente apresentava perda marcante, no que se refere ao bem-estar e à qualidade de vida, com elevado risco de óbito.

Definido o diagnóstico, foi indicada a correção cirúrgica das obstruções anatômicas obstrutivas. Essa conduta constitui a principal recomendação para o correto tratamento e a melhor resposta clínica à síndrome das, e tem por objetivo diminuir a resistência e facilitar o fluxo do ar pelas vias aéreas anteriores (Trappler \& Moore, 2011, Dupré \& Heidenrich, 2016).

Nos braquicéfalos portadores de estenose de narina, a rinoplastia visa à abertura da porção mais anterior da narina, que causa a obstrução, e é considerada uma técnica simples e de fácil execução (Dupré \& Heidenrich, 2016). A palatoplastia tem por finalidade a ressecção da porção caudal do palato mole, impedindo que a obstrução da nasofaringe durante a inspiração e permitindo a melhor passagem do ar para as vias aéreas posteriores (Findji \& Dupré, 2013).

São enumeradas diferentes técnicas cirúrgicas corretivas para a síndrome braquicefálica, podendo ser utilizados eletrocautério bipolar (Brdecka, Rawlings, Perry, Anderson, 2008), eletrocautério monopolar, laser diodo (Dunié-mérigot et al.,2010), bisturi harmônico (Michelsen, 2011), laser de CO2 (Lodato \& mauterer, 2014) e tradicionalmente o bisturi de lâmina fria (Dupré \& Heidenrich, 2016). No atual relato, optou-se pela realização da rinoplastia e da palatoplastia por ablação com laser diodo.

O uso de laser cirúrgico no tratamento da síndrome do braquicefálicos vêm se intensificando (Emmerson, 2014). O emprego desse artifício está ligado diretamente a inúmeras vantagens exibidas em comparação com os procedimentos cirúrgicos convencionais (Dunié-mérigot et al., 2010; Keats, 2012; Lodato \& mauterer, 2014).

O laser de Diodo como o utilizado no procedimento aqui realizado, apresenta um comprimento de onda de $980 \mathrm{~nm}$ com potência variando de 1 a $60 \mathrm{~W}$, com modo de contato oferecendo grande precisão de corte e ablação ou no modo não contato que é bem absorvido por tecidos pigmentados, tendo assim alto poder hemostático (Eeg, 2003). Maior facilidade na execução da técnica foi observado ao se comparar o uso do laser de CO2 com o bisturi de lâmina fria (Davidson et al., 2001) com menos tempo cirúrgico com o uso do laser diodo frente aos outros dois métodos (Dunié-mérigot et al., 2010).

Essas características físicas inerentes aos equipamentos de laser tanto de $\mathrm{CO} 2$, quanto o de diodo são extremamente relevantes pois assim como procedido no atual relato, quando realizada a laser, a rinoplastia e palatoplastia apresentam menores índices de complicação por inflamação pós-operatória, menor tempo anestésico e cirúrgico, menos hemorragia e melhor hemostasia, sem a necessidade da realização de suturas, e melhor controle analgésico trans e pós-operatório, benefícios que garantem maior segurança ao paciente (Core, 2013; Lodato \& Mauterer, 2014).

A rinoplastia com o uso do laser diodo não é muito relatado muito relatado na literatura técnica, os resultados obtidos com as configurações do equipamento ajustadas para o procedimento neste relato justificam o uso do laser de diodo para rinoplastia.

Dunié-mérigot et al., (2010), utilizaram o laser diodo de $980 \mathrm{~nm}$ para palatoplastia no modo de contato pulsado na potência $6 \mathrm{~W}$ e observam edema pós-cirúrgico regional, os autores acreditam que isto ocorreu por danos térmicos colaterais, apesar do uso no modo de contato este fato se reduzir. No relato atual empregou-se o mesmo aparelho no modo de contato contínuo na potência de 3,5w não sendo constatado tal complicação, sugerindo que a aplicação de potência mais baixa no procedimento são eficazes com menores índices de complicação. 
A melhora clínica dos pacientes em resposta ao tratamento cirúrgico deve ser avaliada de forma individual, ponderando-se aspectos como a idade, as alterações anatômicas obstrutivas e a condição geral do animal (Torrez \& Hunt, 2006).

É importante salientar que apesar de bons resultados terapêuticos pós-cirúrgicos serem obtidos por animais adultos ou jovens adultos (Haimel \& Dupré, 2015; Tarricone et al., 2019), como verificado no paciente de 3 anos de idade do presente relato, que após ter sido submetido à rinoplastia e palatoplastia apresentou marcante melhora em sua qualidade de vida, as alterações anatômicas obstrutivas das vias aéreas anteriores devem ser identificadas e corrigidas precocemente para obtenção de melhores resultados (Emmerson, 2014).

Bons resultados, com baixas taxas de complicação, foram observados com o emprego do laser nos procedimentos de rinoplastia e palatoplastia (Lodato, 2012; Ree, Milovancev, MacIntyre \& Townsend, 2016), chegando à média de 96\% de sucesso pós-cirúrgico com alta médica (Tarricone et al., 2019). Em geral, verifica-se marcante melhora clínica dos pacientes a longo prazo, após o tratamento cirúrgico e, consequentemente, melhora no bem-estar e na qualidade de vida dos animais e dos tutores (Lodato, 2012; Ree et al., 2016), conforme relatou o tutor do animal.

\section{Considerações Finais}

São de extrema importância o diagnóstico e o tratamento precoce da síndrome braquicefálica, em função da gravidade e do caráter progressivo da doença, que podem levar o animal a óbito. A técnica de rinoplastia e palatoplastia, com o uso do laser diodo, mostrou-se eficaz no tratamento do paciente, proporcionando melhora na qualidade de vida do animal, além do bom resultado estético obtido.

Consoante aos bons resultados obtidos com o uso do laser de diodo na realização da rinoplastia e palatoplastia, sugere-se que mais estudos sejam realizados mostrando a eficácia desta modalidade no tratamento da síndrome obstrutiva braquicefálica.

\section{Referências}

Brdecka, D. J., Rawlings, C. A., Perry, A. C., \& Anderson, J. R. (2008). Use of an electrothermal, feedback-controlled, bipolar sealing device for resection of the elongated portion of the soft palate in dogs with obstructive upper airway disease. Journal of the American Veterinary Medical Association, 233(8), 12651269 .

Core, D. M. (2013). CO2 laser allows bloodless repair of stenotic nares. Veterinary Practice News, October, 32-33.

Crane, C., Rozanski, E. A., Abelson, A. L., \& de Laforcade, A. (2017). Severe brachycephalic obstructive airway syndrome is associated with hypercoagulability in dogs. Journal of Veterinary Diagnostic Investigation: Official Publication of the American Association of Veterinary Laboratory Diagnosticians, Inc, 29(4), 570-573.

Davidson, E. B., Davis, M. S., Campbell, G. A., Williamson, K. K., Payton, M. E., Healey, T. A., \& Bartels, K. E. (2001). Comparison of CO2 laser and sharp dissection techniques for excision of elongated soft palates in brachycephalic dogs. Proceedings of SPIE, 4244, $575-582$.

De Lorenzi, D., Bertoncello, D., Mantovani, C., \& Bottero, E. (2018). Nasopharyngeal sialoceles in 11 brachycephalic dogs. Veterinary Surgery: VS, 47(3), $431-438$.

Dunié-Mérigot, A., Bouvy, B., \& Poncet, C. (2010). Comparative use of $\mathrm{CO}_{2}$ laser, diode laser and monopolar electrocautery for resection of the soft palate in dogs with brachycephalic airway obstructive syndrome. The Veterinary Record, 167(18), 700-704.

Dupré, G., \& Heidenreich, D. (2016). Brachycephalic syndrome. The Veterinary Clinics of North America. Small Animal Practice, 46(4), 691-707.

Eeg, P. H. (2003). Laser technology offers wide range of surgical applications. DVM in focus, 11, 22-26.

Emmerson, T. (2014). Brachycephalic obstructive airway syndrome: A growing problem. Journal of Small Animal Practice, 55(11), 543-544.

Findji, L., \& Dupre, G. (2013). Brachycephalic Syndrome: Innovative Surgical Techniques. Clinician's brief, 11(6), 79-85.

Haimel, G., \& Dupré, G. (2015). Brachycephalic airway syndrome: A comparative study between pugs and French bulldogs: folded flap palatoplasty in Pugs versus French BD. Journal of Small Animal Practice, 56(12), 714-719.

Keats, M. M. (2012). Brachycephalic airway syndrome, part 1: Correcting stenotic nares. DVM New Magazine, 6S-8S. 
Research, Society and Development, v. 10, n. 2, e33410212630, 2021

(CC BY 4.0) | ISSN 2525-3409 | DOI: http://dx.doi.org/10.33448/rsd-v10i2.12630

Ladlow, J., Liu, N.-C., Kalmar, L., \& Sargan, D. (2018). Brachycephalic obstructive airway syndrome. The Veterinary Record, 182(13), 375-378.

Liu, N.-C., Troconis, E. L., Kalmar, L., Price, D. J., Wright, H. E., Adams, V. J., Sargan, D. R., \& Ladlow, J. F. (2017). Conformational risk factors of brachycephalic obstructive airway syndrome (Boas) in pugs, French bulldogs, and bulldogs. PLOS ONE, 12(8), e0181928.

Lodato, D. L., \& Hedlund, C. S. (2012). Brachycephalic airway syndrome: Management. Compendium (Yardley, PA), 34(8), E4.

Lodato, D., \& Mauterer, J. (2014). Techniques for performing corrective surgery: Dogs with brachycephalic airway syndrome. Today's Veterinary Practice, $34(8), 78-83$.

Michelsen, J. (2011). Use of the harmonic scalpel for soft palate resection in dogs: A series of three cases. Australian Veterinary Journal, 89(12), 511-514.

Oechtering, G. U. (2010). Síndrome braquicefálica - novas informações sobre uma antiga doença congênita. Veterinary Focus, 20(2), 2-8.

Packer, R. M. A., O'Neill, D. G., Fletcher, F., \& Farnworth, M. J. (2019). Great expectations, inconvenient truths, and the paradoxes of the dog-owner relationship for owners of brachycephalic dogs. PLOS ONE, 14(7), e0219918.

Packer, R. M., \& Tivers, M. (2015). Strategies for the management and prevention of conformation-related respiratory disorders in brachycephalic dogs. Veterinary Medicine: Research and Reports, 219.

Pereira, A. S. et al. (2018). Metodologia da pesquisa científica. UFSM.

Pichetto, M., Arrighi, S., Roccabianca, P., \& Romussi, S. (2011). The anatomy of the dog soft palate. II. Histological evaluation of the caudal soft palate in brachycephalic breeds with grade I brachycephalic airway obstructive syndrome. Anatomical Record (Hoboken, N.J.: 2007), 294(7), 1267-1272.

Ree, J. J., Milovancev, M., MacIntyre, L. A., \& Townsend, K. L. (2016). Factors associated with major complications in the short-term postoperative period in dogs undergoing surgery for brachycephalic airway syndrome. The Canadian Veterinary Journal = La Revue Veterinaire Canadienne, 57(9), 976-980.

Sławuta, P., Glińska-Suchocka, K., \& Cekiera, A. (2015). The use of elements of the Stewart model (Strong ion approach) for the diagnostics of respiratory acidosis on the basis of the calculation of a value of a modified anion gap (Agm) in brachycephalic dogs. Polish Journal of Veterinary Sciences, 18(1), 217222.

Tarricone, J., Hayes, G. M., Singh, A., \& Davis, G. (2019). Development and validation of a brachycephalic risk (Brisk) score to predict the risk of complications in dogs presenting for surgical treatment of brachycephalic obstructive airway syndrome. Veterinary Surgery: VS, 48(7), $1253-1261$.

Torrez, C. V., \& Hunt, G. B. (2006). Results of surgical correction of abnormalities associated with brachycephalic airway obstruction syndrome in dogs in Australia. The Journal of Small Animal Practice, 47(3), 150-154.

Trappler, M., \& Moore, K. (2011). Canine brachycephalic airway syndrome: Surgical management. Compendium (Yardley, PA), 33(5), E1-7; quiz E8 